ArtefaCToS. Revista de estudios de la ciencia y la tecnología

eISSN: $1989-3612$

Vol. 9, No. 1 (2020), 2. a Época, 77-99

DOI: https://dx.doi.org/10.14201/art2020917799

\title{
Filosofía de la innovación y valores sociales en las empresas
}

\section{Philosophy of Innovation and Social Values in Enterprises}

\section{Javier ECHEVERRÍA}

Jakiunde, España

javierecheverria@jakiunde.org

Recibido: 27/03/2020. Revisado: 01/04/2020. Aceptado: 05/04/2020

\section{Resumen}

La teoría schumpeteriana de la innovación y de los empresarios innovadores (entrepreneurs) sigue teniendo validez en los mercados. Generalizando algunas definiciones de Schumpeter es posible desarrollar una filosofía de la innovación que valga para diversos tipos de innovación, incluidas las innovaciones sociales y los descubrimientos científicos. En este artículo se propone un enfoque axiológico en estudios de innovación y, siguiendo al Stanford Center for Social Innovation, se definen las innovaciones sociales como aquellos procesos sociales que generan valor social. Se comentan algunas definiciones recientes del valor social y se propone una definición heurística general: las innovaciones son procesos interactivos que generan algo nuevo, transformador y valioso en entornos y sistemas determinados. Para medir el valor social, se opta por el modelo poliédrico, basado en stakeholders (Retolaza y San-José, 2016). Dicha metodología permite integrar valores sociales contrapuestos y, por tanto, posibilita el estudio de barreras y oposiciones a las propuestas socialmente innovadoras, tanto si surgen en las empresas y en los mercados como en las sociedades en general.

Palabras clave: Estudios de innovación; Filosofía de la innovación; Gestión de stakeholders; Axiología; Valor social; Entrepreneurs; Descubrimiento científico.

\begin{abstract}
The Schumpeterian theory of innovation and innovative entrepreneurs is still valid in the markets. By generalizing some definitions of Schumpeter it is possible to develop a philosophy of innovation that could be valid for various
\end{abstract}

Ediciones Universidad de Salamanca / @ब

ArtefaCToS, Vol. 9, No. 1 (2020), 2. á́poca, 77-99 
types of innovation, including social innovations and scientific discoveries. This article proposes an axiological approach in innovation studies and, following the Stanford Center for Social Innovation, social innovations are defined as those social processes that generate social value. Some recent definitions of social value are commented on and a general heuristic definition is proposed: innovations are interactive processes that generate something new, transformative and valuable in certain environments and systems. To measure the social value, we choose the polyhedral model, based on stakeholders (Retolaza and San-José, 2016). This methodology allows the integration of conflicting social values and, therefore, enables the study of barriers and oppositions to socially innovative proposals, whether they arise in companies and markets or in societies in general.

Keywords: Innovation studies; Philosophy of Innovation; Stakeholder management; Axiology; Social Value; Entrepreneurs; Scientific Discovery.

\section{Schumpeter y los empresarios innovadores}

Schumpeter reconoció que las innovaciones se producen en diversos tipos de sociedades (Schumpeter, 1986, 222), pero subrayó que caracterizan a las economías competitivas y de libre mercado, donde los empresarios innovadores (entrepreneurs) son el motor del crecimiento económico. Según él:

el empresario y su función no son difíciles de conceptualizar: la característica definitoria es simplemente el hacer nuevas cosas o el hacer de una manera nueva cosas que ya se habían hecho. (Schumpeter, 1986, 223)

Schumpeter centró, pues, su teoría de la innovación en las acciones empresariales, en la medida en que generan valor económico. Pero ya en 1909 había hablado de valor social (Schumpeter 1909). Asimismo definió el emprendizaje sobre la base de la acción, no a las ideas: hacer nuevas cosas y hacer cosas de una manera nueva. Obsérvese que no son aportaciones hechas de pasada: estaba intentado definir el emprendizaje y la innovación. En este artículo se parte de esa "definición schumpeteriana" del concepto de innovación, que formulamos así: la innovación consiste en hacer nuevas cosas o en hacer cosas que ya se habian hecho de una manera nueva. En el caso de las empresas, hay que analizar las acciones empresariales y la cadena de valor (Porter) en cuyo contexto actúan los emprendedores.

Desarrollando la concepción de Schumpeter, el principal canon de los actuales estudios de innovación, el Manual de Oslo (OCDE, 1992), ha distinguido cuatro tipos de innovación: de producto, de proceso, organizacionales y comunicacionales $o$ de marketing. Hacer algo nuevo conlleva una innovación de producto, mientras hacer las cosas de una manera nueva caracterizaría a las innovaciones de proceso. Pero en el caso de acciones colectivas, como son las actividades empresariales, intervienen varios agentes, siendo importante cómo se organizan las 
empresas y qué relaciones tienen con sus proveedores, distribuidores, clientes y usuarios, pero también con los reguladores, sin olvidar a los competidores. Por eso es preciso recurrir al modelo sistémico (Lundvall, 1992; Nelson 1993), que afirma que los procesos de innovación no sólo se desarrollan en las empresas, sino en los sistemas locales, regionales y nacionales de innovación, que es donde las empresas innovadoras actúan. Los economistas, sociólogos y politólogos que sentaron las bases del Manual de Oslo y aplicaron el modelo sistémico en teoría de la innovación, fueron considerados como neoschumpeterianos: las innovaciones no sólo proceden de los empresarios individuales, sino que emergen en los sistemas nacionales, regionales y locales de innovación, generando importantes cambios en los mercados y sociedades correspondientes. Precisamente por ello, además de generar un valor económico y alterar el equilibrio de los mercados, los emprendedores generan valor social, que, sin embargo, es más difícil de precisar y contabilizar que el valor monetario de las acciones empresariales, el cual se mide en las cuentas analíticas de resultados anuales.

Si en lugar de pensar únicamente en el mundo de los negocios nos referimos a los seres humanos en general, una filosofía de la innovación que pretenda ser válida en el siglo XXI debe ir más allá de la economía y ha de incluir una teoría de las nuevas acciones humanas y de los nuevos modos de hacer. Las TICs (tecnologías de la información y la comunicación) aportan un ejemplo muy claro, puesto que además de generar nuevos objetos digitales, han traído consigo nuevos modos de hacer cosas, incluyendo nuevos modos de hacer negocios y generar beneficios económicos y sociales. Su irrupción y propagación global ha sido claramente disruptiva, tanto en los mercados como en las sociedades. En este artículo nos referiremos ante todo al valor económico y social generado por las empresas digitales, pero también por los usuarios de dichas tecnologías, puesto que los usuarios también pueden ser agentes innovadores (von Hippel, 2005).

La definición schumpeteriana recién mencionada puede ser matizada y mejorada desde una perspectiva filosófica. Lo importante son las acciones y actividades innovadoras, así como los agentes que promueven dichas actividades y las agendas que utilizan para desarrollarlas. En principio se trata de los empresarios, pero el modelo sistémico de Lundvall y Nelson dejó claro que, para que haya innovaciones, se requiere el concurso de otros agentes para que las propuestas innovadoras tengan éxito, incluida la difusión y la mayor o menor aceptación social de dichas propuestas. Surgió así la teoría de los stakeholders (Freeman, 1984), que en este artículo ampliamos a los procesos de innovación social, en los que también hay diversos agentes involucrados y afectados. Las propuestas socialmente innovadoras también pueden ser aceptadas o rechazadas. Siempre hay que tener en cuenta las reacciones o resistencias a la innovación (barreras), no sólo los agentes innovadores (emprendedores). Quienes compiten con las empresas innovadoras, quienes trabajan en ellas, quienes distribuyen sus productos e incluso sus clientes y usuarios también están involucrados en los procesos de innovación y deben ser estudiados en tanto stakeholders de los mismos, sean o no empresarios. Como 
afirmó Rogers a mediados de los años 60 y aceptó luego el Manual de Oslo, sin difusión no hay innovación. Las innovaciones tecnológicas y empresariales tienen una dimensión económica que se manifiesta en los mercados, pero también tienen una dimensión social, en la medida en que transforman las acciones de los clientes y usuarios, los cuales no son pasivos ante las innovaciones. Los estudios de innovación han de tener en cuenta a los diversos stakeholders, no sólo a los empresarios. La aceptación y la difusión social de los nuevos productos, y en general de las nuevas acciones, es clave para que un proceso de innovación tenga éxito. Precisamente por ello los nuevos productos y los nuevos procesos, aun teniendo un valor mercantil, tienen también un valor social. Otro tanto puede ocurrir en el caso de las actividades científicas, en las cuales también hay científicos emprendedores e innovadores, lo cual es importante en el contexto de descubrimiento.

Desde una perspectiva filosófica general no hay que limitarse al valor económico y al valor social de las innovaciones. Cabe hablar incluso de generación de valor epistémico en en el caso de las acciones científicas. Generalizando las propuestas de Schumpeter, diremos que una concepción filosófica de la innovación ha de estar basada en los haceres y modos de hacer, en la categoría de novedad y en la noción de valor, la cual admite varias acepciones. Los descubrimientos y avances científicos generan valor en la empresa cientifica, puesto que aportan nuevos conocimientos y nuevos modos de hacer ciencia.

Para mostrar hasta qué punto este enfoque axiológico de la innovación amplía dicho concepto pondré un ejemplo matemático, que surgió en Grecia: el problema de la cuadratura del círculo. Muchos matemáticos intentaron resolverlo positivamente. Demostrar que no es posible cuadrar el círculo con regla y compás fue una de las grandes aportaciones de la matemática del siglo XVII. Por tanto, supuso una innovación matemática de primer orden, porque aportó un conocimiento radicalmente nuevo. Además, trajo consigo un nuevo modo de hacer matemático, sin regla ni compás: ni más ni menos que el método de las series de Wallis y Newton y el cálculo diferencial e integral de Leibniz. Lo mismo cabría decir del teorema de Gödel o de la reciente demostración de Wiles del teorema de Fermat. Una demostración matemática es innovadora cuando consigue demostrar algo que ningún matemático había conseguido demostrar antes o cuando demuestra algo ya conocido mediante otro procedimiento. Demostrar algo ya demostrado mediante un nuevo procedimiento, hasta entonces desconocido, conlleva una innovación matemática de proceso. En ambos casos se produce algo nuevo, pero también valioso, a saber: epistémicamente valioso. Aunque el economista austríaco sólo pensaba en la economía y en los mercados al hablar de innovación, la filosofía puede generalizar sus propuestas y aplicarlas a las matemáticas y a otras muchas ciencias, así como a las nuevas tecnologías. Podemos distinguir entre un experimento que prueba un hecho nuevo y un experimento que comprueba un hecho ya conocido, pero lo hace de una manera nueva: con otros aparatos, siguiendo otros procedimientos, en otros contextos empíricos, en condiciones más generales, etc. La distinción entre innovación de producto y de proceso es útil 
en filosofía de la ciencia, lo mismo que las innovaciones organizacionales (crear la Academia de Platón, el Liceo de Aristóteles, las Universidades Medievales, las Sociedades Científicas de la modernidad o las Politécnicas del siglo XIX) y las comunicacionales (publicar en revistas de impacto, hoy en día).

El propio Schumpeter subrayó el interés de esta primera definición de innovación en los términos siguientes: "es una ventaja que esta definición no establezca una línea definida entre lo que es y no es "empresa", porque la vida real no conoce una división tan definida" (Schumpeter, 1986, 223). El concepto de empresario (entrepreneur, emprendedor) puede generalizarse, y con él la noción de innovación. Cuando un científico individual o un grupo investigador acomete la tarea de hacer un nuevo experimento que permite descubrir algún nuevo hecho científico que modifica el conocimiento previamente aceptado, ha tenido lugar una innovación cientifica. De esta manera, la noción de descubrimiento científico se relaciona con la de innovación, hasta el punto de que cabe hablar de un contexto de innovación en ciencia, no sólo de descubrimiento. Conclusión: tanto en el caso de las ciencias formales como en el de las ciencias físico-naturales, se pueden establecer conexiones estrechas entre las teorías del cambio científico y las teorías de la innovación científica. La distinción clásica en filosofía de la ciencia entre contexto de descubrimiento y contexto de justificación puede ser replanteada en términos de innovaciones exitosas o fracasadas.

Hemos puesto el ejemplo de las actividades científicas, pero las consideraciones precedentes pueden ser ampliadas a otros haceres humanos, incluyendo las acciones sociales, culturales, artísticas, jurídicas, políticas, militares y, por qué no, también medioambientales. Por eso proponemos la siguiente caracterización filosófica general de la innovación:

Def. 1: Innovar es hacer algo nuevo y valioso, o hacer algo que ya se hacía, pero de una manera nueva y valiosa.

Esta definición aporta un primer punto de partida para definir en general el concepto de innovación y, por tanto, para una posible filosofía de la innovación.

En otro pasaje, Schumpeter $(1986,222)$ añadió tres requisitos al concepto de innovación:

a. La emergencia de una innovación no puede ser predicha a partir de los datos pre-existentes.

b. Una innovación cambia el curso ulterior de los acontecimientos, incluso a largo plazo.

c. Las acciones innovadoras proceden de personas, y en particular de individuos. 
El requisito a) tiene gran importancia para los estudios de innovación, puesto que afirma que los procesos de innovación tienen lugar en condiciones de incertidumbre. El requisito b) también es relevante, por dos motivos. En primer lugar, porque de cualquier innovación se derivan consecuencias favorables y desfavorables. Ello implica una concepción consecuencialista de la innovación, en la que no sólo se tienen en cuenta los resultados inmediatos, sino también sus consecuencias ulteriores. En segundo lugar, porque algunas innovaciones no sólo aportan cambios, sino transformaciones profundas. Tal es el caso de las innovaciones disruptivas, que son las que más interesaron a Schumpeter. De hecho, él siempre insistió en que una innovación auténtica rompe el equilibrio de los mercados y por eso llegó a decir que las innovaciones son destrucciones creativas. Este segundo requisito puede incorporarse a la definición, en cuyo caso no sólo hablaríamos de acciones nuevas y valiosas, sino de acciones nuevas y valiosas que transforman el ámbito o sistema donde se desarrollan. Novedad, valor y transformación pasan a ser los tres requisitos básicos en filosofía de la innovación.

En cuanto al requisito c), plantea uno de los temas principales para los estudios de innovación: supuesto que las innovaciones se refieren a nuevas acciones, ¿quiénes son los agentes que las promueven? Pueden ser individuales, como afirmó Schumpeter, pero también colectivos, por ejemplo empresas, ciudades, regiones o incluso sociedades innovadoras. Schumpeter fue un individualista metodológico convencido, no en vano fue quien inventó la expresión "individualismo metodológico". Ahora bien: aunque haya individuos innovadores, también hay que contemplar la existencia de agentes innovadores colectivos, por ejemplo cuando se trabaja en grupo o en instituciones y empresas innovadoras. En suma: hay agentes innovadores individuales, colectivos e institucionales; pero también hay barreras individuales, colectivas e institucionales a la innovación. Para analizar los procesos de innovación hay que tener en cuenta a todos los stakeholders, tanto a los favorables como a los desfavorables. Esta es la propuesta principal del presente artículo. Las innovaciones generan valor para algunos agentes, pero también disvalor para otros. Otro tanto sucede en el caso del valor social que generan las empresas. Para algunos grupos sociales puede ser positivo, y por eso aceptan los nuevos productos. Pero también puede haber oposición y rechazo social (disvalor social). Los estudios de innovación han de ocuparse de lo positivo y de lo negativo, y por eso es preciso el enfoque basado en una pluralidad de perspectivas o stakeholders.

Schumpeter añadió un cuarto comentario, que también es importante en filosofía de la innovación: "debería verse en seguida que lo nuevo no necesita ser espectacular o de importancia histórica; no es preciso que sea el acero Bessemer o el motor de explosión; puede ser una salchicha Deerfoot" (Schumpeter, 1986, 223).

Dicho en nuestros propios términos: las innovaciones pueden producirse a escala micro-, meso- o macro-. Hay innovaciones que sólo transforman los microcosmos, pero las transformaciones que inducen en ellos pueden ser radicales: 
valgan como ejemplo las obras de remodelación de una casa, o la mudanza a un nuevo piso, a un nuevo barrio o a una nueva ciudad. Para quienes experimentan un cambio así, su Lebenswelt se transforma radicalmente: aparecen nuevos espacios de acción y nuevos modos de hacer las cosas cotidianas: nuevas costumbres, y por tanto nuevos hábitos. La filosofía de la innovación que aquí se propugna se ocupan de diversos tipos de valores (mercantiles, tecnológicos, empresariales, epistémicos, sociales y culturales, incluidos los valores morales), pero también de distintas escalas espaciales y temporales donde se desarrollan dichos procesos de innovación.

Consideradas en su conjunto, las cuatro matizaciones que Schumpeter hizo a su "primera definición" son dignas de consideración. Surge así una "segunda definición" de innovación, inspirada en Schumpeter, aunque él no la formuló:

Def. 2: Las innovaciones son acciones (y maneras de hacer) nuevas que generan transformaciones valiosas a escala macro, micro o meso.

En el caso de Schumpeter, en primera instancia se transforman los mercados económicos, sea mediante nuevos productos, nuevos procesos, nuevas formas de organización, nuevos mercados o nuevas materias primas. Una innovación de ruptura ha de tener efectos visibles en algún sector económico y esos resultados serán positivos para unos y negativos para otros, puesto que innovar implica destrucción creativa. En suma: los resultados de un proceso de innovación pueden ser valiosos para unos stakeholders y disvaliosos para otros. Llegamos así al punto clave del enfoque axiológico que propugnamos en este artículo: lo nuevo, en la medida en que sea transformador, admite diversas valoraciones, no todas coincidentes.

\section{Generación de valor en los procesos de innovación}

Por su parte, otro clásico en los estudios de innovación, el sociólogo inglés Everett Rogers, añadió que no hay innovaciones sin difusión de las mismas. Eso suele lograrse a través de los mercados, pero también por diversos circuitos de comunicación social (hoy en día Internet y las redes sociales). En un proceso de innovación hay que distinguir como mínimo tres fases: la invención, la innovación propiamente dicha y la difusión ulterior de dicha innovación a mayor o menor escala. En función de dicho ámbito de difusión las innovaciones son locales, regionales, nacionales o internacionales (globales, hoy en día). Los estudios de innovación han de ser escalables. No es lo mismo analizar un proceso de innovación a nivel local que otro que afecta a diferentes países y culturas, en cuyo caso resulta imprescindible disponer de métodos para comparar y homologar los datos disponibles. Además, esos datos han de ser agregables. El Manual de Oslo fue elaborado para satisfacer todo esos requisitos, varios de los cuales van más allá de Schumpeter. 
Ahora bien: el éxito mayor o menor de una innovación se mide en la fase de difusión, no en la de invención. Supuesto que existiera un equilibrio previo en el mercado, las innovaciones lo romperán, generando pérdidas para unos y ganancias para otros:

Los efectos de la actividad empresarial afectan a la totalidad del sistema, dislocando valores y disrumpiendo el equilibrio antes existente: el término Vendaval expresa correctamente los efectos de dichas ganancias y pérdidas. (Schumpeter, 1939, 135)

Esta transmutación de valores se produce en los mercados y se manifiesta en forma de ganancias para unas empresas y pérdidas para otras. Para ilustrar su teoría Schumpeter puso muchos ejemplos empíricos, como el mercado de la seda cuando se inventó el nylon. Llegó a la conclusión de que la innovación es discontinua y procede por "explosiones": "conlleva una alteración (disturbance) de las estructuras existentes y opera más como una serie de explosiones que como una transformación amable, aunque incesante" (Schumpeter, 1939, 100). Esta es una de las aportaciones características de Schumpeter: la disruptividad. Los actuales estudios de innovación, sin embargo, se ocupan también de los procesos de innovación acumulativa.

En cualquier caso, a lo largo de un proceso de innovación se genera riqueza económica, pero también se destruye. Hay ganadores y perdedores. Es preciso analizar los procesos de innovación por su capacidad de generar y destruir valor, no sólo por su novedad. Por nuestra parte, aceptamos plenamente que los procesos de innovación, cuando son exitosos, generan valor, sea éste económico o de otro tipo. La generación de valor es la propiedad características de la innovación, para nuestro enfoque axiológico. Esto nos lleva a considerar una tercera definición:

Def. 3: Las innovaciones son acciones (y maneras de hacer) nuevas, que transforman $X$ y generan valor a partir de $X$, sea a escala macro, micro o meso.

Esta tercera definición todavía tiene insuficiencias. En primer lugar, no subraya suficientemente la condición procesual de la innovación, que implica la intervención de varios agentes en un determinado entorno espacio-temporal. Por otra parte, tampoco distingue entre novedad e innovación, siendo así que no toda nueva acción conlleva innovaciones. Se requiere que dichas acciones, o sus resultados, sean valoradas positivamente, por ejemplo en los mercados, y en general en las sociedades. En tercer lugar, el Manual de Oslo, siendo neoschumpeteriano, parte de la hipótesis de que quienes innovan son los empresarios. Conviene ampliar esa perspectiva, retomando la idea schumpeteriana originaria, que dejaba suficientemente vaga la noción de empresarios (entrepreneurship), como anteriormente hemos mostrado. En cuarto lugar, muchas innovaciones no tienen éxito. Es importante tener presente este hecho. Muchos ideólogos de la inno- 
vación afirman que sólo es innovación aquello que tiene éxito en los mercados. No es así. Las ideas innovadoras han fracasado muchísimas veces a lo largo de la historia. Como dice Chesbrough $(2011,23)$, "la mayoría de las innovaciones fracasan, y las compañías que no innovan desaparecen”. Conclusión metodológica: hay que prestar atención a las innovaciones fallidas, no sólo a las exitosas. Aprender de los propios fracasos es una de las cualidades de las personas innovadoras. En quinto lugar, un agente innovador crea valor, pero también destruye valor. Un empresario innovador puede hacer mucho daño a otras empresas e incluso llevarlas al cierre por no ser competitivas, con las consecuencias subsiguientes para trabajadores y accionistas. Los procesos de innovación empresarial en los mercados libres implican creación y destrucción. En sexto lugar, no sólo hay diferentes tipos de innovación, sino también diferentes escalas. Este hecho es muy importante para los estudios de innovación, puesto que la metodología cambiará en función de los casos de estudio: innovación local, urbana, regional, nacional, global; o también innovación a escala macro-, meso- y micro-.

Por todo ello, proponemos una nueva definición, donde la condición procesual y sistémica de nuestra filosofía de la innovación quede clara. Es la siguiente:

Definición heurística propuesta: "Las innovaciones son procesos interactivos que generan algo nuevo, transformador y valioso en entornos y sistemas determinados (a escala macro-, meso- o micro)" (Echeverría, 2017, 82).

Esta definición engloba las tres definiciones anteriores, así como las consideraciones de Schumpeter y las de otros autores, incluyendo las definiciones antes mencionadas de innovación social. Por tanto, nos permite investigar diversos procesos de innovación social, y a diversas escalas, siempre desde un enfoque axiológico: como generación (y destrucción) de valor. Será la que usemos en el resto del presente artículo, puesto que sintetiza la propuesta filosófica que hacemos. Damos, pues, por establecidas las bases iniciales y provisionales para seguir desarrollando una filosofía de la innovación. No se trata de una definición que pretenda precisar la esencia de la noción de innovación, sino que tiene una función heurística, por aportar una caracterización suficientemente general y, a nuestro modo de ver, fecunda.

\section{Innovación social como generación de valor social}

Los estudios de innovación social surgieron en Canadá y en el Reino Unido a principios del siglo XXI (Crises, Young Foundation, NESTA) y se expandieron rápidamente por los países industrialmente desarrollados. Las políticas para impulsar la innovación social pasaron a formar parte de la agenda europea, sobre todo a partir de 2010. Para impulsarlas, la Comisión Europea asumió tres o cuatro definiciones de innovación social, que se han convertido en canónicas para politólogos y consultores. 
Las más difundidas fueron propuestas por la Young Foundation británica, el NESTA (National Endowment for Science, Tecnology and Arts) y la asociacion mundial SIX (Social Innovation Exchange) y han tenido considerable aceptación. Según la Young Foundation, las innovaciones sociales son:

actividades y servicios innovadores que surgen con el objetivo de satisfacer una necesidad social y que predominantemente son desarrolladas y difundidas a través de organizaciones cuyos principales propósitos son sociales. (Young Foundation, 2007, 7)

Consecuentemente con esta definición, en la que se habla explícitamente de organizaciones, centraron sus estudios y análisis en las organizaciones, fuesen gubernamentales o no, prestando muy poca atención a las innovaciones sociales promovidas por personas concretas o por movimientos sociales, a pesar de que en principio aceptaban que los individuos también pueden ser socialmente innovadores. La Young Foundation, el NESTA y, por ampliación, el Gobierno británico, prestaron particular atención a las empresas sociales, incluidas aquellas con programas de responsabilidad social corporativa. Sin embargo, Mulgan y sus colaboradores opusieron en 2007 la innovación social a la business innovation, cuyo objetivo principal estriba en obtener beneficios económicos y que es llevada a cabo por empresas con afán de lucro. A partir de 2006, el NESTA emitió importantes informes sobre innovación para el gobierno británico, algunos de los cuales tuvieron gran influencia en las políticas británicas de innovación. Los gobiernos de Tony Blair y Gordon Brown llegaron a encargarle la elaboración de un sistema de indicadores de innovación diferente al Manual de Oslo, el Nation Innovation Index (2008), donde la innovación social estuvo contemplada, a diferencia de la OCDE, que se ocupó exclusivamente de la innovación empresarial y tecnológica. De esta manera, la innovación social pasó a formar parte de las políticas públicas de innovación, y también de las estrategias de algunas empresas privadas. Ese marco conceptual fue aceptado por otros países anglosajones (Australia, Nueva Zelanda) y el BEPA europeo lo acabó adoptando en buena medida a partir del documento Empowering People (2010), que ha orientado los programas europeos de innovación social durante la década 2010-2020.

En 2010, el NESTA y la red SIX publicaron el Study on Social Innovation en el que propusieron una definición más general, basada en la distinción entre medios y fines:

Las innovaciones sociales son innovaciones que son sociales en sus fines y en sus medios. (SIX y NESTA 2010, 17-18)

Si a esta definición le añadimos que los agentes que promueven las innovaciones sociales son, ante todo, organizaciones, queda perfilado el paradigma anglosajón en estudios y políticas de innovación, en lo que atańe a la innovación social. Sin embargo, esa definición tiene el inconveniente de asumir la concep- 
ción instrumental de la racionalidad, basada en la distinción entre medios y fines. Como resultado, se presupone asimismo que los procesos de innovación social tienen una intencionalidad, cosa que a que veces sucede, pero por lo general no. Por nuestra parte, a la racionalidad instrumental le contraponemos la racionalidad axiológica (Agazzi, Rescher), según la cual tanto las acciones como las interacciones humanas y sociales están guiadas por valores, y normalmente por valores contrapuestos entre los diversos stakeholders intervinientes en un proceso de innovación. En dichos procesos, que suelen transcurrir en condiciones de incertidumbre, nos parece preferible hablar de valores, puesto que cada agente interviniente no sólo valora los medios que elige para actuar, sino también las metas y los objetivos de sus acciones. Por otra parte, en muchos procesos sociales los agentes tienen intenciones y metas distintas, lo cual no impide que surjan innovaciones sociales como resultado de sus interacciones en contextos determinados. Las innovaciones sociales pueden ser propuestas por agentes y organizaciones concretas, pero su difusión y desarrollo suele ser muy compleja, interviniendo múltiples factores y objetivos contrapuestos, por lo que, cuando tienen éxito, no son resultado de las intenciones de sus agentes promotores, sino de los valores y del grado de aceptación social que encuentran entre grupos sociales muy diversos (stakeholders sociales). En particular, las innovaciones sociales disruptivas rompen el statu quo social y benefician a unos grupos y agentes, perjudicando a otros. Por tanto, es erróneo atribuir una intencionalidad social generalizada y compartida por los agentes sociales, aunque en ocasiones pueda haberla. Conclusión: para ampliar la disruptividad schumpeteriana a otros tipos de valores, es preciso reemplazar la racionalidad instrumental por una racionalidad axiológica, que incluye valores parcialmente compartidos, pero también valores opuestos. La racionalidad instrumental presupone que, para elegir los medios más eficientes para lograr un fin, dicha finalidad ha de estar dada y debe ser compartida por los diversos agentes sociales intervinientes, algo que raras veces ocurre en las interacciones sociales, y menos en las innovaciones disruptivas. El enfoque axiológico, en cambio, permite estudiar innovaciones mixtas de muy diversos tipos: económicas, sociales, tecnológicas, empresariales, políticas, militares, jurídicas, administrativas y medioambientales. Los procesos de innovación pueden generan valor y disvalor tanto si cumplen los objetivos iniciales de sus agentes promotores como si no. Esto vale, en particular, para las fases de difusión, aceptación y apropiación de las propuestas innovadoras. Una cosa son los fines de las organizaciones innovadoras y otra muy distinta las razones por las que los usuarios de productos y servicios sociales, por ejemplo, utilizan dichos productos y servicios.

Como previamente había mostrado von Hippel, la innovación de usuarios es clave en muchos sectores económicos, y también sociales (von Hippel, 2005). Los objetivos de los usuarios y consumidores al actuar pueden ser muy distintos a los objetivos y metas de las organizaciones que proponen innovaciones, sean éstas tecnológicas o sociales. Un ejemplo típico, muy estudiado por von Hippel, fue la utilización de los teléfonos móviles como instrumentos para la escritura, y 
no sólo para hablar a distancia, que era como los fabricantes los habían diseñado históricamente. La conversión de los teléfonos móviles en artefactos para escribir (y hoy en día para adjuntarse todo tipo de mensajes, incluidas imágenes y videos) fue una innovación disruptiva de usuarios, que los fabricantes tuvieron que hacer suya. Por eso hay que atender a las interacciones entre los diversos stakeholders y no sólo a las metas y objetivos que cada cual puede tener en un proceso de innovación multiagente. Medir el valor social generado por una empresa es una tarea a llevar a cabo teniendo en cuenta a los diversos stakeholders, no sólo a los inversores, promotores y gestores de la propuesta innovadora. Si optamos por analizar una pluralidad de agentes involucrados, no hay objetivos ni fines comunes a todos ellos, salvo en situaciones muy concretas. Resumimos esta opción diciendo que nuestro enfoque, además de axiológico, es pluralista. Hay que tener siempre en cuenta una pluralidad de valores, incluida la combinación de valores empresariales y sociales en las acciones innovadoras de una organización.

\section{Innovación social como creación de valor y disvalor social}

La filosofía de la innovación que proponemos afirma que la innovación es un valor, que se vincula a sistemas de valores que guían las acciones humanas. No excluye la posibilidad de que diversos agentes compartan objetivos y fines, pero amplía el campo de estudio a procesos interactivos en los que intervienen diversos agentes con intereses, fines y valores diferentes. La ventaja principal del enfoque axiológico consiste en que, siendo los valores funciones que orientan las acciones humanas, es posible componer funciones, y por ende valores, incluidos valores contrapuestos. Cada agente no consigue sus objetivos, porque otros stakeholders actúan en direcciones diferentes, pero sí logra una satisfacción parcial de sus criterios de valoración, hasta un grado que considera suficiente. Esta racionalidad axiológica, ampliamente investigada por los seguidores de la teoría de la racionalidad acotada de Herbert Simon, resulta clave para los estudios de innovación social, sobre todo si hablemos de valores sociales, y no sólo de necesidades, demandas o fines sociales, es decir: de una racionalidad axiológica acotada.

Por eso comentaremos en el presente apartado la aportación que desde 2003 hizo a los estudios de innovación social la Universidad de Stanford. Ese ańo comenzó a editar la revista electrónica Stanford Social Innovation Review, en cuyo primer número se definió la innovación social como "el proceso de inventar, dar soporte seguro e implementar nuevas soluciones a necesidades y problemas sociales" (Phills, Deiglmeier, y Miller, 2008, 1). Se pasaba así de la noción de necesidad social a la de problema social, lo cual supuso una ampliación conceptual muy positiva, a nuestro juicio. Cuando se inventan nuevas soluciones a problemas sociales previamente existentes (o sobrevenidos) y, además, se les busca soporte e implementación, entonces surgen los procesos de innovación social. En ese mismo escrito fundacional el Stanford Center for Social Innovation señaló que la innovación social puede surgir de los tres sectores, el público, el privado y el 
que no tiene ánimo de lucro (non profit sector). Las empresas, las administraciones y la propia sociedad civil pueden promover innovaciones sociales.

Retomando esas ideas germinales, el Director de dicho Centro, James A. Phills, junto con dos investigadores del mismo, Kriss Deiglmeier y Dale T. Miller, publicaron en 2008 un artículo importante, en el que distinguieron entre innovación social, emprendizaje social (social entrepreneurship) y empresa social (social enterprise). A modo de ejemplo, Phills, Deiglmeier y Miller afirmaron que los microcréditos que promovieron el Premio Nobel de la Paz Muhammad Yunus y el Grameen Bank en la India constituyen la quintaesencia de la innovación social, porque afrontan de forma novedosa uno de los principales problemas sociales en aquel país, la pobreza. También pusieron otros ejemplos relacionados con el sector financiero, dejando claro su interés por las finanzas sociales, uno de los ámbitos donde la sensibilidad y la responsabilidad social de algunos empresarios pueden llegar a ser patentes, contrariamente a otros, que sólo piensan en maximizar al valor y la rentabilidad económica de sus inversiones. Un empresario o un inversor que, sin renunciar a obtener beneficios económicos, acepta que éstos no sean tan elevados como hubiera sido posible, porque sus acciones e inversiones resuelven además problemas sociales, son agentes que funcionan conforme a la racionalidad axiológica acotada, combinando valores empresariales y financieros con valores sociales, de modo que los primeros no sean los únicos a tener en cuenta.

Phills, Deiglmeier y Miller asignaron el concepto de empresa social a las organizaciones, y definieron a los emprendedores sociales como personas concretas con determinadas cualidades: "audacia, responsabilidad, ingenio, ambición, persistencia y asunción de riesgos" (Phills et al., 2008, 2). Dijeron que ambos tipos de agentes, tanto los individuos innovadores como las organizaciones colectivas, surgen ante todo del non profit sector. Pero su aportación más importante, a nuestro modo de ver, consistió en proponer la noción de valor social y afirmar decididamente que "en último término, innovación social es lo que crea valor social” (Phills et al., 2008, 2). Según ellos, los estudios de innovación estudian en general la creación de valor, y los de innovación social el valor social. Ahora bien, ¿cómo definir este último?

Phills, Deiglmeier y Miller comentaron cuatro acepciones diferentes. La primera dependía de las intenciones de los agentes. La segunda distinguía los valores en función de sus campos de aplicación, siendo el valor social creado ante todo por las organizaciones no gubernamentales y sin ánimo de lucro. La tercera acepción se centraba en las necesidades y problemas que preocupan a una sociedad en su conjunto y que, caso de solucionarse, generan valor social. La cuarta acepción oponía los valores sociales a los valores económicos y financieros. Lo notable es que consiguieron conjugar esas cuatro acepciones en una misma definición, según la cual el valor social implica "la creación de beneficios o la reducción de costes para una sociedad mediante esfuerzos por resolver necesidades y proble- 
mas sociales mediante vías que van más allá de las ganancias privadas y de los beneficios generales que se derivan de las actividades mercantiles" (Phills et al., 2008, 2).

Así pues, dieron por supuesto que tanto las actividades que provienen del sector público como las que generan los mercados son socialmente beneficiosas. A continuación, refirieron el valor social a la satisfacción de necesidades y a la resolución de problemas sociales. Para ello, aunque distinguieron entre valor social y valor económico, definieron el primero sobre la base de la relación coste social/ beneficio social. Dichas aportaciones son muy interesantes desde una perspectiva axiológica porque relacionan los procesos de innovación con la generación de valor e introducen las nociones de beneficio social y coste social. Sin embargo, son mejorables en algunos puntos, como luego veremos.

Los tres investigadores del Stanford Center for Social Innovation propusieron finalmente dos definiciones de innovación social. La más completa es la siguiente:

Una solución nueva a un problema social que es más efectiva, más eficiente, más sostenible y más justa que las soluciones ya existentes, y para la cual el valor creado corresponde principalmente a la sociedad como un todo más que a los particulares (Phills et al., 2008, 2).

Esta definición es interesante filosóficamente porque conjuga las nociones de novedad, valor y solución/problema. Sus núcleos conceptuales iniciales son la noción de novedad y la dicotomía solución/problema. Además, añade el requisito de que las nuevas soluciones han de ser preferibles a las anteriormente existentes. Y para decidir sobre esa preferencia aportan cuatro criterios de valoración: efectividad, eficiencia, sostenibilidad y justicia.

No queda claro por qué eligieron estos cuatro criterios de valoración y no otros a la hora de comparar soluciones a problemas sociales. Los cuatro son relevantes, sin duda, pero puede haber otros valores sociales a tener en cuenta, por ejemplo la inclusión, la solidaridad o la responsabilidad, por mencionar tres valores sociales particularmente importantes, entre otros que podrían mencionarse, como la igualdad de oportunidades. Aun sin argumentar del todo su propuesta, Phills, Deiglmeier y Miller dieron un paso muy importante para el enfoque axiológico que aquí propugnamos. Esta se sitúa claramente en el terreno de los valores, no en el de los fines ni los medios.

Nuestra objeción principal atañe a la exigencia final, según la cual el incremento de valor afecta a la sociedad considerada como un todo ("as a whole"). La mayoría de las innovaciones sociales pueden beneficiar a grupos sociales concretos, no a toda la sociedad. Ese requisito final, por tanto, nos parece demasiado restrictivo. En cualquier caso, Phills, Deiglmeier y Miller vincularon claramente su teoría de la innovación social a la creación de valor social, incluyendo la suge- 
rencia de que una innovación social ha de traer consigo algún incremento en el grado de satisfacción de ciertos valores sociales concretos. La novedad por sí sola no basta: se requiere además una valoración positiva, y por tanto una mejora. Dicha mejora conlleva una cambio en el statu quo, y por ende una transformación social, que será más o menos grande, según estemos analizando microcosmos, mesocosmos o macrocosmos sociales. Una definición así permite analizar pequeñas innovaciones sociales, que son promovidas por personas en microcosmos concretos, no por organizaciones.

A nuestro modo de ver, Phils, Deiglmeier y Miller aportaron un buen punto de partida para una posible filosofía de la innovación que se ocupe de la innovación social en términos de generación de valor social. Por nuestra parte, interpretamos su aportación de la manera siguiente:

a. Una novedad es algo relativo a una situación anterior, en la que existían tales o cuales soluciones propuestas. Por tanto, los estudios de innovación siempre son situados y han de tener en cuenta los estados anteriores del arte, no sólo los resultados que se obtengan.

b. Para que una innovación sea aceptada es preciso que haya sido valorada positivamente e, incluso, que lo haya sido en mayor grado que las soluciones anteriores. Por tanto, lo nuevo ha de resultar preferible a lo anterior, al menos para determinados stakeholders.

c. Las innovaciones sociales implican creación de valor social, mientras que otras modalidades de innovación generan o incrementan otras modalidades de valor. Por tanto, los estudios de innovación deben partir de una axiología.

d. Las innovaciones sociales provienen de cualquiera de los tres sectores (privado, público, tercer sector), y en particular de la colaboración entre los tres.

e. Las innovaciones sociales, como las innovaciones en general, son procesos que se desarrollan a lo largo del tiempo, pero también pueden ser consideradas como los resultados que se derivan de dichos procesos. Esta doble faz de la innovación ha de ser tenida en cuenta. No sólo los resultados finales pueden generar valor (como afirman los tres autores de Stanford), sino también las demás fases del proceso. La condición procesual de la innovación es clave para una filosofía de la innovación, porque nos lleva a una ontología de procesos, no de cosas, objetos y sujetos.

f. Asimismo aceptamos la hipótesis de que hay varios tipos de innovaciones, no sólo innovaciones de producto. Al respecto, las propuestas de los autores de Stanford pueden ser implementadas por las del Manual de Oslo. Por tanto, puede haber innovaciones sociales de producto, de proceso, organizativas y de marketing (o comunicación). 
Estos seis puntos aportan otras tantas bases conceptuales para una filosofía de la innovación con enfoque axiológico, pluralista y acotado. En todo caso, la innovación social implica la emergencia de un nuevo paradigma, muy distinto al de la innovación tecnológica y empresarial, porque está basado en otros valores.

\section{Medir el valor social de las actividades empresariales}

El problema difícil de solucionar es la estimación del valor social, y en su caso su medición. Para orientar políticas y estrategias de innovación social es preciso disponer de datos cuantitativos. En el caso de la innovación social, ello no es fácil, como muchos autores han subrayado. Geoff Mulgan, por ejemplo, publicó en 2010 un artículo en la Stanford Social Innovation Review en el que afirmó taxativamente que hay cientos de métodos para medir el valor social (Mulgan, 2010 , 2) y que ninguno de ellos es satisfactorio, por una razón principal: casi todas las métricas disponibles suponen que la noción de valor es algo objetivo y, según Mulgan, no lo es, ni siquiera en el caso del valor económico: "como la mayoría de economistas están ahora de acuerdo, el valor no es un hecho objetivo; en lugar de eso, el valor emerge de la interacción entre oferta y demanda, y en último término refleja lo que la gente o las organizaciones están dispuestos a pagar" (Mulgan, 2010, 3).

Curiosamente, para argumentar esta tesis Mulgan no aportó una empresa como ejemplo, sino una administración pública, el servicio británico de salud (NHS), cuyo valor social había sido medido por la Young Foundation por encargo del Gobierno británico (Mulgan, 2010). No vamos a comentar aquí ese informe en detalle. Nos limitaremos a señalar que, desde el principio, su planteamiento fue muy reduccionista. Dio por supuesto que el valor social sólo puede ser medido en términos monetarios, sobre la base de los costes que los servicios sociales pueden tener en el "mercado de las empresas sociales". Sin embargo, como veremos a continuación, hay métodos económicos para medir el valor social que genera una organización sin reducir lo social a lo económico y, en particular, sin presuponer la existencia de un "mercado de empresas sociales", noción esta muy problemática, pues ni siquiera suele haber un listado fiable de empresas sociales en un país o región, ni mucho menos una "Bolsa de empresas sociales" donde se establezca el valor económico de cada una de ellas. Una expresión así sólo tiene un sentido metafórico, por ejemplo cuando una administración pública decide repartir recursos económicos para financiar proyectos socialmente innovadores y publica una convocatoria al respecto, como han solido hacer la Young Foundation, el NESTA y el propio BEPA europeo. Pero ocurre que las innovaciones sociales no sólo surgen de las administraciones o empresas, sino también de la propia sociedad civil. Muchas de ellas surgen para afrontar problemas sociales con los medios disponibles, que suelen ser recursos humanos y medios sociales muy limitados, que sin embargo son capaces de afrontar y resolver problemas sociales planteados a su escala. Por ejemplo: la solidaridad de una población ante una 
catástrofe natural, o la de una persona como Yunus o una empresa financiera social como el Grameen Bank ante situaciones de extrema pobreza en la India. De hecho, muchas de las innovaciones sociales son sobrevenidas. Aparecen por sorpresa y provienen de usuarios y ciudadanos. En todo caso, son esas innovaciones, cuando tienen éxito, las que luego generan "mercados de empresas sociales". Éstos no preexisten a los emprendedores sociales.

En el fondo, aunque en este artículo no vayamos a argumentarlo con detalle, una filosofía de la innovación que asume la definición heurística antes mencionada puede aplicar esa misma definición a la propia noción de precio y afirmar que el valor de mercado de un determinado producto o servicio surge porque dichos productos o servicios tienen un valor de uso para muchas personas, las cuales interactúan entre sí hasta conformar un sistema de precios por dichos productos o servicios. Los precios surgen por la interacción entre ofertantes y demandantes en espacios singulares específicos a los que tradicionalmente se ha denominado mercados y ferias, y, como tal emergencia o punto de equilibrio entre oferta y demanda, pueden ser considerados como auténticas innovaciones socio-económicas, puesto que aportan un valor de mercado económico, pero también un valor de uso social, a partir del cual surgen los precios en los mercados locales y regionales. Mulgan tiene razón al señalar que los precios son entidades relacionales que surgen a partir de oferta y demanda, y que fluctúan con éstas, por ser variables a lo largo del tiempo, pero también según los lugares y regiones donde se organicen dichos mercados. De hecho, los precios son muy distintos en unos y otros mercados locales. Por tanto, podemos mantener la hipótesis de que, desde la perspectiva de la filosofía de la innovación, los precios de productos y servicios en un mercado son el resultado de procesos interactivos que aportan algo nuevo, transformador y valioso en dichos entornos económicos. Dichos entornos y sistemas, insistimos en ello, no solo son mercantiles, sino también sociales, puesto que dichos objetos y servicios tienen valor de uso y no sólo valor de cambio. En suma: la propia noción de precio sería una innovación socio-económica, desde nuestro punto de vista axiológico, relacional y emergentista.

Argumentar esta propuesta nos llevaría muy lejos. Nos limitamos a plantearla y, para terminar, mencionaremos una metodología de medición del valor social que han propuesto desde 2012 dos grupos de investigadores que colaboran estrechamente, uno liderado por José Luis Retolaza en la Universidad de Deusto (Business School), y otro por Leire San-José en la Universidad del País Vasco (Facultad de Económicas de Sarriko). Al marco conceptual del que parten lo han llamado modelo poliédrico y está basado en la existencia de diversos stakelholders en los procesos de innovación social, los cuales puede ser empresas sociales, administraciones públicas u organizaciones del tercer sector (no gubernamentales). Dicho modelo encaja bien con nuestros planteamientos y aporta procedimientos precisos para calcular el valor social de un programa llevado a cabo por una organizacion. Todavía más: monetiza el valor social, pero sin reduccionismo alguno. 
Esa monetización es un proxy y un indicador del valor social que atribuyen los diversos stakeholders a una determinada iniciativa o acción innovadora.

Resumiremos esa metodología siguiendo el hilo de algunas de las aportaciones que han hecho Retolaza, San-José, Ruiz-Roqueñi y Gartzia (2015, 2016 y 2019, por ejemplo) en las que afirman la posibilidad de desarrollar una contabilidad social en las organizaciones partiendo de los siguientes planteamientos teóricos: "Tradicionalmente, el enfoque de análisis económico-financiero en las organizaciones se ha centrado en el valor que las empresas generan para sus accionistas, de manera que el indicador de valor básico ha sido el beneficio después de intereses e impuestos" (Gartzia y Retolaza, 2019, 19). Sin embargo, estos investigadores han puesto en consideración la "necesidad de ampliar la perspectiva en la generación de valor de los accionistas al conjunto de los grupos de interés" (Freeman, 1984; Retolaza y San-José, 2016; San-José y Retolaza, 2016) y para ello manifiestan "la necesidad de considerar un concepto más amplio de valor, que sea capaz de integrar el valor económico y el valor social" (Gartzia y Retolaza, 2019, 19).

Las empresas y organizaciones disponen así de un instrumento, el modelo poliédrico, que mide el valor social integral generado por ellas, parte del cual es económico, y se manifiesta en las cuentas anuales de resultados, pero habiendo otra parte social, que ha de ser medida por una contabilidad social de nuevo cuño:

La propuesta de una contabilidad social orientada a la sostenibilidad tiene por objetivo cuantificar en unidades monetarias el Valor Social Integral que generan, o destruyen, las diversas organizaciones; y cuenta con tres propuestas complementarias: 1) de una parte, incorpora un modelo subyacente de valor, el Modelo poliédrico, centrado en la Teoría de Stakeholder. Se trata de un modelo teórico, y por lo tanto sujeto a debate conceptual. 2) En segundo lugar, se ha desarrollado una metodología procesual, basada en el mencionado modelo poliédrico, que a través de una serie de pasos permite sistematizar el proceso de cálculo de valor social para cada entidad particular; esta metodología, a la que se suele referir como SPOLY (Social Polyhedral Model) o como VSI (Valor Social Integrado), puede y debe ser mejorada de forma constante a través del feedback proporcionado por su aplicación en diferentes organizaciones. 3) En tercer lugar, tiende a generarse una estandarización sectorial, tanto de las variables de valor utilizadas en el análisis del valor de no-mercado como de los proxis que permiten la cuantificación en unidades monetarias de los outputs con ellas vinculados. (Gartzia y Retolaza, 2019, 19)

Vamos a comentar únicamente el primer punto, porque es el más importante para la filosofía de la innovación, dado que atañe a la noción de valor económico-social, o socio-económico. Ese valor integral o mixto se articula en el ya mencionado modelo poliédrico, que suele ser presentado de la manera siguiente: 


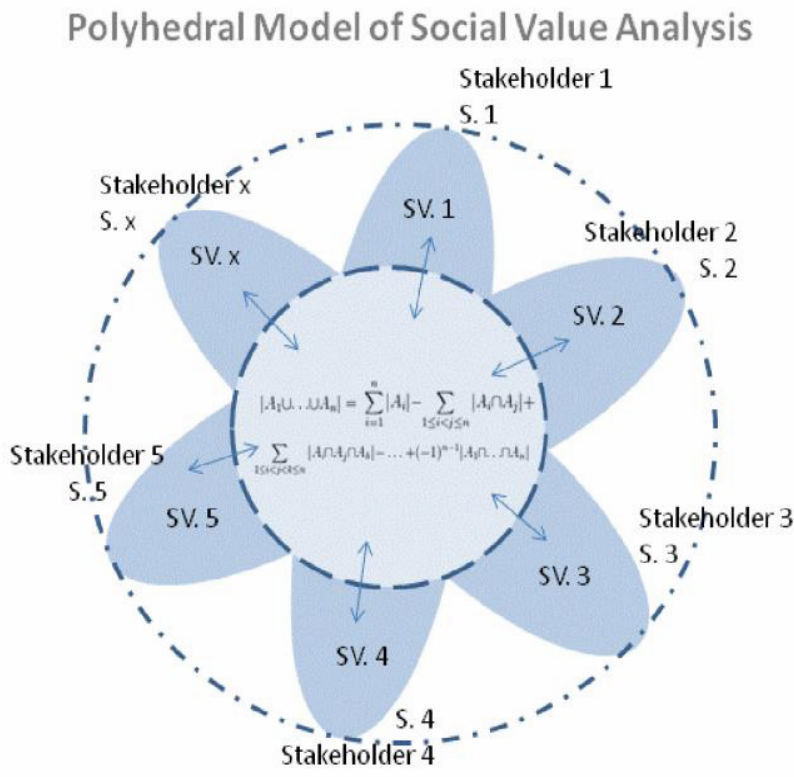

Figura 1. Modelo poliédrico de valor social. Fuente: Tomado de Retolaza, San-José \& Ruiz-Roqueñi, 2016, 40.

La figura muestra que es posible medir el valor social que cinco o más agentes involucrados en un proceso de innovación atribuyen a las acciones que conforman dicho proceso, algunas de las cuales pueden ser valiosas económicamente, y otras socialmente. Cada stakeholder hace su propia valoración de los resultados, y ello desde su propia perspectiva, que puede ser muy distinta e incluso opuesta a la de los agentes que promovieron la innovación e iniciaron el proceso. La clave del modelo poliédrico consiste en que, aun siendo diferentes esas valoraciones, puede existir un núcleo de valor común a los diversos agentes involucrados o afectados por el proceso innovador. A dicho núcleo, representado en la figura por el círculo, se le denomina valor compartido (shared value). Agrupa variables de valor que en ocasiones ni siquiera son tenidas en cuenta por los agentes, puesto que estos piensan ante todo en sus objetivos y fines más inmediatos, sin tener presente otros valores concomitantes:

Las diferentes áreas representan el valor generado (SV) para cada uno de los stakeholder (Stakeholder $\mathrm{n}^{\circ} \mathrm{X}$ ); dichos valores no tienen por qué ser coincidentes, lo normal es que en algunas variables coincidan, y en otras no. El núcleo central representa el conjunto de valor atribuido a las variables coincidentes, lo que podríamos denominar como shared value; el cual se calculará mediante el sumatorio de los valores coincidentes para el conjunto de stakeholders. Complementariamente existirán valores generados para un stakeholder particular, que no sean coincidentes con los de los otros stakeholders. La consolidación del total del valor generado por la organización para el conjunto de stakeholders constituirá el valor integral generado. (Gartzia y Retolaza, 2019, 21). 
Dicho valor integrado es más amplio y complejo que el valor propiamente económico. De hecho, el modelo poliédrico tiene en cuenta incluso a agentes que no están inicialmente involucrados en dicho proceso, pero cuyas consecuencias ulteriores sí les pueden afectar. También a esos "non stakeholders" hay que tenerlos en cuenta, y preguntarles si estiman positiva o negativamente el producto, proceso o servicio innovador en curso. Por tanto, el modelo poliédrico es claramente pluralista y perspectivista. Conjuga e integra en un mismo modelo de medición diferentes valoraciones, e incluso valoraciones opuestas, lo cual es particularmente novedoso.

Este último aspecto del modelo poliédrico resulta importante para nuestro enfoque, en el que se asume que puede haber valores y actitudes contrapuestas entre los diversos agentes que interactúan en un proceso de innovación, sea éste empresarial o social. Incluso podríamos expandirlo y aplicar el modelo poliédrico a los descubrimientos e innovaciones científicas y tecnológicas, en la medida en que tengan valor social. Algunas de ellas, en efecto, encuentran inicialmente rechazo, sobre todo cuando son disruptivas, porque implican un cambio de paradigma en las teorías científicas admitidas. Por otra parte, no hay que olvidar que la tecnociencia contemporánea tiene una dimensión empresarial significativa, tanto a la hora de captar recursos económicos mediante proyectos y contratos, como luego al gestionar los recursos humanos y al publicitar los resultados obtenidos. Las tecnociencias generan conocimientos, y por ende valor epistémico; pero también suelen generar valor económico (a través de las patentes resultantes, por ejemplo, o simplemente por el volumen de presupuestos e inversiones manejados en una investigación, cuya gestión requiere modelos empresariales), así como valor social, el cual en algunos casos es positivo, porque la línea de investigación tiene aceptación social, pero en otros casos también hay valoraciones negativas, e incluso rechazos por parte de sectores sociales significativos, los cuales son stakeholders. La ingeniería genética y los alimentos transgénicos son un ejemplo canónico: aportan innovaciones, pero su valor social puede ser a veces negativo, dependiendo de los diversos criterios de valoración de los stakeholders sociales.

Esta es la razón por la que, en el presente artículo, hemos subrayado la existencia de valoraciones diferentes, e incluso contrapuestas. A la hora de medir el valor social de un avance tecnocientífico no basta con los indicadores de valor epistémico (impactos en revistas del primer decil, por ejemplo), aunque tanto la novedad como el valor del conocimiento aportado sean importantes. Adicionalmente, hay que medir el coste económico de un proyecto o programa de investigación tecnocientífica, así como sus beneficios y sus costes sociales. Normalmente habrá unos y otros, a juicio de los diversos stakeholders.

Lo importante es que el modelo poliédrico aporta una metodología precisa para abordar todas estas cuestiones, incluso en el caso de discrepancias valorativas significativas. A diferencia de otros procedimientos usados para determinar 
el valor social, dicho modelo es pluralista y está basado en la existencia de una variedad de stakeholders en cualquier proceso de innovación, y en general en las actividades empresariales. De hecho, las empresas competidoras siempre se ven afectadas por una determinada innovación, sea de producto o de proceso.

Esta es una de las peculiaridades de los estudios de innovación, a los que no cabe aplicar la racionalidad instrumental de medios y fines, salvo en fases y momentos muy concretos. La incertidumbre respecto a los resultados que habrá y los modos de valorarlos es una de las grandes peculiaridades de la filosofía de la innovación, a diferencia de la filosofía de la ciencia, donde la resolución de problemas propuestos suele ser relativamente predecible, incluyendo aquellos casos, tan importantes en la historia de la ciencia, en los que se demuestra que tal o cual problema científico es irresoluble con tales o cuales medios. En suma: los estudios de innovación pueden inspirarse en los estudios de ciencia y tecnología, y ello tanto en los estudios estrictamente epistemológicos como en las dimensiones sociales de la ciencia y la tecnología (enfoque CTS). Pero las condiciones de incertidumbre en las que emergen y se desarrollan las innovaciones acarrean dificultades específicas, debido a que la generación de valor social es un proceso complejo, en el que intervienen diversos agentes, con intereses, objetivos y valores contrapuestos. Por eso es preciso utilizar modelos axiológicos pluralistas y basados en stakeholders a la hora de medir el valor social que tienen las actividades y empresas tecnocientíficas. Valga un ejemplo obvio: las innovaciones militares que generan armas de destrucción masiva. Si son usadas contra poblaciones civiles, su valor social será negativo y el rechazo muy amplio, por muy positivo que sea su valor militar. Sólo apoyarán su construcción y uso aquellas sociedades o sectores sociales a quienes la guerra pudiera favorecer. El resto estará en contra, o a lo sumo se declarará neutral. No hay que olvidar que hoy en día hay empresas tecnocientíficas militares muy poderosas ni que también existe el emprendizaje militar, por ejemplo el terrorismo, aunque no suela ser estudiado por los estudios de innovación ni por el Manual de Oslo. La condición destructiva de muchas innovaciones resulta particularmente clara en esos casos, sin perjuicio de que las armas puedan tener un valor positivo para los ejércitos y las sociedades que deciden empuñarlas. En estos casos, el valor social de este emprendizaje siempre es parcial. Nunca favorece a toda la sociedad, por la sencilla razón de que estamos ante sociedades enfrentadas. En tales situaciones, el valor social lo aportan los diplomáticos, los negociadores y quienes atienden a las víctimas y a los refugiados. Y los emprendizajes militares abundan en el siglo XXI, aunque no suelan ser estudiados ni tengamos datos fiables sobre ellos. 


\section{A modo de conclusión}

Desde una perspectiva filosófica basada en el pluralismo y en la racionalidad axiológica acotada, hay que optar por la generación de valores mixtos (blended values), en lugar de pensar que el valor económico y el valor social son variables independientes. De hecho, ambas modalidades de valor están muy correlacionadas. Por eso hay que hablar de crear valor socio-económico, y no sólo económico ni monetario. Asimismo hay que tener en cuenta que, por su condición procesual, las innovaciones involucran a diversos agentes, no sólo a los proponentes de las innovaciones. A partir de ello, la definición heurística antes mencionada y el enfoque axiológico ofrecen un marco adecuado para interpretar muchas innovaciones empresariales que generan valor mixto. Incluso hay metodologías económicas no reduccionistas que permiten calcular el valor social integrado de las actividades realizadas por una empresa innovadora, como hemos resaltado en el último apartado. Todo ello abre un campo de investigación que merece la pena explorar.

\section{Referencias bibliográficas}

BEPA (2010). Empowering people, driving change: Social innovation in the European Union. Report prepared by Agnès Hubert et alia. Bruxelles: European Commission, Bureau of European Policy Advisers.

Chesbrough, H. W. (2011). Innovación abierta. Barcelona: Plataforma.

Cloutier, Julie (2003). Qu'est-ce que l'innovation sociale? Quebec: Cahiers de Crise.

Echeverria, Javier (2014), Innovation and Values: a European Perspective, translated by Cameron J. Watson. Reno (NV): University of Nevada at Reno, Center for Basque Studies.

Echeverría, Javier (2017), El arte de innovar. Naturalezas, lenguajes, sociedades. Madrid: Plaza y Valdés.

Freeman R. Edward (1984). Strategic management: a stakeholder approach. Boston: Pitman.

Gartzia, Leire y Retolaza, José Luis (2019). Cuantificación en unidades monetarias del valor social del género en las organizaciones. Vitoria-Gazteiz: Emakunde, Informe del Instituto Vasco de la Mujer.

Lundvall, Bengt-A. (1992). National systems of Innovation: Towards a theory of interactive learning. London: Pinter.

Mulgan, Geoff (2007a). Social Innovation: what is it, why it matters, how it can be accelerated. London: Young Foundation.

Mulgan, Geoff (2007b). Ready or Not: Taking innovation in the Public Sector Seriously. London: Young Foundation. 
Nelson, Richard R. (1993). National Innovations Systems: a Comparative Analysis. Urbana, Ill.: University of Urbana-Champaign.

NESTA (2009). The Innovation Index. Measuring the UK's investment in innovation and its effects. London: NESTA Index Report.

Phills, James A. Jr., Kris Deiglmeier, \& Dale T. Miller (2008). Rediscovering Social Innovation. Stanford Social Innovation Review, Fall 2008.

OECD (1992). OECD Proposed Guidelines for Collecting and Interpreting Technological Innovation Data: Oslo Manual. Paris: OECD.

Retolaza, José Luis, San-José, Leire y Ruiz-Roqueñi, M. (2016). Social Accounting for Sustainability. Monetizing the Social Value. Heidelberg: Springer.

Rescher, Nicholas (1999). On Situating Process Philosophy. Process Studies, 28(1/2), $37-42$.

Rogers, Everett K. (1995). Diffusion of Innovations. New York: The Free Press, $4^{\text {th }}$ edition.

San-José, Leire y Retolaza, José Luis (2016). Contabilidad social orientada a los stakeholders. Madrid: Pirámide.

Schumpeter, Joseph A. (1909). On the Concept of Social Value. Quarterly Journal of Economics, 23, 213-232.

Schumpeter, Joseph A. (1939). Business Cycles. New York: McGraw-Hill 1939.

Schumpeter, Joseph A. (1942). Capitalism, Socialism and Democracy. New York: Harper \& Row, ed. 1975.

Schumpeter, Joseph A. (1986). Ensayos. Barcelona: Oikos-Tau.

SIX and Young Foundation (2010). Study on Social Innovation. Bruxelles: European Union/Young Foundation.

Von Hippel, Eric (2005). Democratizing Innovation. Harvard, Mass.: Harvard University Press. 
